\title{
Trastornos de la conducta alimentaria, experiencias adversas vitales e imagen corporal: Una revisión sistemática
}

\author{
Ester Idini ${ }^{1}$, Alberto Barceló-Soler ${ }^{2}$, Mayte Navarro-Gili ${ }^{3}$, Pamela Paredes-Carreño ${ }^{1}$, \\ Daniel Pérez-Tausia ${ }^{4}$ y Javier García-Campayo ${ }^{1,2}$
}

${ }^{1}$ Hospital Universitario Miguel Servet, Zaragoza, España

${ }^{2}$ Instituto de Investigación Sanitaria Aragón, Zaragoza, España

${ }^{3}$ Universidad de Zaragoza, Zaragoza, España

${ }^{4}$ Hospital Royo de Vilanova, Zaragoza, España

Eating disorders, life stressful events and body image:

A systematic review

\begin{abstract}
Our objective was to clarify the relationship between adverse life events and the altered body image in eating disorders. We reviewed English and Spanish papers indexed in MEDLINE, PsycINFO and ScienceDirect from the last 10 years. We included 16 studies of which $37.5 \%$ focus on eating disorders. Sexual abuse, physical abuse, domestic violence, bullying and having experienced multiples adverse life events have been associated with overweight patients and high body dissatisfaction. Emotional abuse has been associated with underweight and body shape concerns. Posttraumatic symptoms and emotion dysregulation have been identified as mediating factors. Eating disorder psychopathology could be the response to a bad regulation of negative emotion coming from different adverse life events and this mechanism could underlie patiens' body dissatisfaction.
\end{abstract}

Keywords: eating disorders; adverse life events; bulimia nervosa; anorexia nervosa; binge-eating disorder; body image; body dissatisfaction; traumatic events.

Resumen: El objetivo de este trabajo es esclarecer la relación entre experiencias adversas vitales y la alteración de la imagen corporal en los trastornos alimentarios. Se han revisado trabajos en inglés y castellano indexados en MEDLINE, PsychoINFO y ScienceDirect en los últimos 10 años. Se han incluido 16 estudios de los cuales, el $37.5 \%$ se centra en trastornos de la alimentación. El abuso sexual, el físico, la violencia intrafamiliar, el acoso escolar y la suma de diferentes experiencias adversas vitales identifican pacientes con sobrepeso y alta insatisfacción corporal. El abuso emocional se ha asociado a bajo peso y preocupación entorno a la figura. La relación con la imagen corporal está mediada por clínica postraumática y la desregulación emocional. La clínica alimentaria es una respuesta a un mal manejo de emociones negativas derivadas de diferentes experiencias adversas y este mecanismo podría estar en la base de la insatisfacción corporal de los pacientes.

Palabras clave: trastornos de la alimentación; experiencias adversas vitales; bulimia nerviosa; anorexia nerviosa; trastorno de atracón; imagen corporal; insatisfacción corporal; eventos traumáticos.

Recibido: 23 de agosto de 2020; aceptado: 15 de marzo de 2021. Correspondencia: Ester Idini, Departamento de Psiquiatría, Hospital Miguel Servet, Zaragoza. Paseo Isabel la católica 1-3, 50009 Zaragoza, España. Correo-e: eidini@salud.aragon.es.
Agradecimientos: Manifestamos nuestro agradecimiento al Grupo Aragonés de Investigación en Salud mental en Atención Primaria-B76-Gob. de Aragón. 


\section{Introducción}

Desde que se describió el primer caso moderno de anorexia nerviosa (Lasegue, 1873), los trastornos de la conducta alimentaria (TCA) han ido cobrando protagonismo por el aumento de su incidencia, su morbi-mortalidad y la aparición a edades cada vez más tempranas, así como en la edad adulta (Micali et al., 2017; Treasure et al., 2020). Se trata de enfermedades psiquiátricas que se explican desde un modelo biopsicosocial, habiéndose observado un cambio en la presentación fenomenológica a lo largo de los años (Treasure et al., 2018). En el manual diagnóstico DSM-5 (American Psychiatric Association, 2014) se encuentran, dentro del epígrafe TCA, la anorexia nerviosa con sus variantes restrictiva, purgativa y mixta, la bulimia nerviosa, el trastorno por atracón, la pica, el trastorno de la rumiación, la anorexia nerviosa atípica, la bulimia subclínica, el trastorno por atracón subclínico y el TCA no especificado.

En su presentación fenomenológica, al debut de la enfermedad, se describen cambios en los hábitos alimentarios, que pueden ir desde la restricción de las comidas hasta la presencia de ingesta compulsiva asociada a vomito postprandial, en un intento de controlar el peso. La autoestima de estos pacientes suele estar excesivamente influenciada por la apariencia, presentando habitualmente una alta insatisfacción corporal (Treasure et al., 2020); además suelen presentar una alteración del estado de ánimo, conductas impulsivas de riesgo como tendencia autolesiva, y comorbilidad con otras patologías psiquiátricas (Treasure et al., 2020). Actualmente, representan un alto coste para los servicios sanitarios y un sufrimiento para los pacientes y sus familias (Wildes y Marcus, 2013; Wufong et al., 2019). Entre los factores que influyen en el desarrollo y curso del trastorno encontramos la ansiedad, la depresión, la presencia de rasgos obsesivos compulsivos, como el perfeccionismo, la baja autoestima, el aislamiento social y la dificultad en la regulación emocional (Anderluh et al., 2003; Egan et al., 2013; Treasure et al., 2020). Respecto al tratamiento, las principales dificultades son el retraso en el diagnóstico, la comorbilidad psiquiátrica, el bajo peso, la adicción a tóxicos y la presencia de complicaciones médicas (NICE, 2017). A pesar de una correcta implementación de los tratamientos psicológicos solo se recuperan un $50 \%$ de las personas, y del resto, el $30 \%$ mejora parcialmente, mientras que el $20 \%$ se cronifica presentando deterioro social y laboral (Helverskov et al., 2010; NICE, 2017). En los pacientes más resistentes se han descrito altos niveles de preocupación por el peso y la figura corporal (Marco et al., 2004). Dicha preocupación entorno al cuerpo y la representación que tienen los pacientes de su figura es una de las principales responsables de las recaídas tras la recuperación ponderal y psicopatológica (Keel et al., 2005; Tabri et al., 2015) y uno de los aspectos más difíciles de tratar con los abordajes actualmente presentes (Vaquero-Cristóbal et al., 2013; Cook-Cottone, 2015; Paxton y McLean, 2010; Sepúlveda et al., 2002).

En la construcción de la identidad corporal confluyen factores cognitivos, afectivos, neurológicos y sociales (Shilder, 1935; Rosen, 1992; van den Berg et al., 2007; Quick y Byrd-Bredbenner, 2014; Tylka, y Wood-Barcalow, 2015). Se distingue, por un lado, el «esquema corporal» que se organiza a nivel cerebral a partir de percepciones táctiles, visuales, espaciales y nos permite estimar adecuadamente nuestra silueta y, por otro lado, la «imagen corporal», constructo más complejo al confluir en él deseos, necesidades, emociones, cogniciones y relaciones con otras personas (De Vignemont, 2009). La magnificación/sobreestimación de la silueta es lo que define la distorsión de la imagen corporal que, según algún estudio en anorexia nerviosa (Grunwald et al., 2002) depende de la alteración del córtex parietal derecho, mientras que las actitudes y pensamientos negativos hacia el cuerpo determinan nuestra insatisfacción corporal (Hayaki et al., 2002). Aunque la preocupación entorno al cuerpo no es patognomónica de los trastornos alimentarios, en estos pacientes la distorsión de la imagen y la insatisfacción corporal asumen una dimensión más compleja por como representan, sienten y viven su cuerpo que se transforma en un enemigo portador de sensaciones displacenteras (González-Carrascosa et al., 2013; Salaberria et al., 2007; Lethbridge et al., 2011; Bedoya-Hernández y Marín-Cortés, 2010).

En las últimas dos décadas, en psiquiatría se ha aceptado la presencia del estrés como factor implicado en la etiopatogenia de las enfermedades mentales (Herbert, 1997). Se puede definir el estrés como el conjunto de adaptaciones a nivel neuroendocrino e inmunológico que el organismo pone en marcha frente a cualquier evento que vive como amenazante. Diferentes estudios han evidenciado como el estrés psicosocial que se asocia a experiencias adversas vitales se relaciona con una mayor morbi-mortalidad tanto de enfermedades mentales, como de otras patologías médicas (Trucco, 2002; Devi et al., 2019). Entre las diferentes experiencias que se generan en las etapas de la infancia, adolescencia y adultez que se han asociado al desarrollo y evolución de los TCA (Carretero-Garcia et al., 2012; Molendijk et al., 2017) se cuentan las que derivan de situaciones impersonales como desastres naturales (incendios, terremotos), $\mathrm{y}$ las que se generan en las relaciones interpersonales sea con extraños (guerras, violaciones, hurtos, agresio- 
nes...) o dentro de las relaciones de apego, describiéndose en los diferentes estudios, el abuso emocional que implica la humillación y las críticas, el abuso físico cuando hay agresiones, el abuso sexual, la negligencia emocional que implica el rechazo, la negligencia física cuando no se atiende a las necesidades básicas, y la violencia sexual entre otros (Kira et al., 2008).

En TCA se han reportado prevalencias de experiencias adversas vitales que van desde un 5\% hasta un $66 \%$ (Neumark-Sztainer et al., 2000; Fosse y Holen, 2006). Además de los eventos descritos, se han evaluado accidentes de tráfico, pérdida de seres queridos, violencia de género, riesgo de muerte por enfermedad grave y acoso escolar como situaciones generadoras de daño (Striegel-Moore et al., 2002; Lejonklou et al., 2014; Striegel-Moore y Bulik, 2007), observándose, en los últimos años, un aumento importante de estudios realizados sobre experiencias adversas y su relación con la gravedad de la clínica alimentaria (Caslini et al., 2016; Pignatelli et al., 2017; Trottier y MacDonald, 2017). Todavía no hay datos suficientes para que se consideren factores pronósticos de la enfermedad, pero se han identificado posibles mediadores asociados que empeoran el trastorno, y que podrían ser diana de tratamiento, como la clínica postraumática (Isooma et al., 2015; Malinauskiene y Malinauska, 2018), la desregulación emocional (Mills et al., 2015) y las experiencias disociativas (Moulton et al., 2015; Palmisano et al, 2018). Algún autor sugiere la asociación entre experiencias adversas vitales y una construcción negativa del self a través del desarrollo de una afectividad negativa (Schore, 2003). El modelo transdiagnóstico de los TCA, elaborado por Fairburn et al. (2003), señala como la alteración de la imagen corporal es una de las características común a todas las categorías diagnosticas. Sin embargo, los estudios centrados en la relación entre experiencias adversas vitales y la identidad corporal son pocos frecuentes. Los encontrados, más que analizar su relación con la distorsión de la imagen corporal se centran en evaluar el tipo de asociación con el peso y la insatisfacción corporal, siendo los resultados bastantes controvertidos (Treurer et al., 2005; Taylor, et al., 2006). Diferentes estudios han evaluado la asociación entre el tipo específico de situación potencialmente traumática y la alteración de la imagen corporal, siendo la violencia sexual, el abuso emocional y el físico asociados a una mayor insatisfacción corporal sea en población sin diagnóstico clínico que en pacientes con TCA (Kenardy y Ball, 1998; Grilo y Masheb, 2001; van Gerko et al., 2005; Preti, et al., 2006).

Considerando que la insatisfacción corporal es un factor perpetuador del TCA, y la distorsión de la imagen un elemento psicopatológico clave de este tipo de tras- tornos, unido al aumento de la incidencia, su alta morbimortalidad, así como su escasa respuesta a los tratamientos, se estima relevante profundizar en el conocimiento de cómo las experiencias adversas vitales contribuyen en el desarrollo de la imagen corporal. Hasta donde alcanza nuestro conocimiento, no hemos encontrado ninguna revisión reciente sobre el tema. Nuestra hipótesis es que, tras vivenciar experiencias traumáticas sobre todo en una edad vulnerable los pacientes desarrollan una sensación de inseguridad y, al no poder controlar los eventos derivados de la experiencia, crean una falsa sensación de seguridad a través del control del cuerpo y la comida. Creemos que la insatisfacción corporal de las pacientes deriva de un mal manejo de las emociones negativas ligadas a la experiencia adversa vital que se traduce en afectividad negativa asociada a la alteración de la imagen corporal. Expuesto lo anterior, el objetivo de este trabajo ha sido revisar y sintetizar de forma crítica los estudios publicados en los último 10 años sobre experiencias adversas vitales, TCA e imagen corporal para esclarecer el tipo de relación que existe entre dichas experiencias y la imagen corporal. La pregunta que queremos contestar es si la relación que existe entre las experiencias aversas vitales y la imagen corporal, es directa como ya se describió en algún estudio (Madowitz et al., 2015) o es determinada por posibles mediadores que podrían representar diana de tratamiento.

\section{Método}

Esta revisión se desarrolló siguiendo las pautas y orientaciones de la guía PRISMA (Moher et al., 2015).

\section{Búsqueda y fuentes de información}

Realizamos una búsqueda inicial en las bases de datos MEDLINE, PsycINFO y ScienceDirect. En todas las bases se introdujeron las 4 secuencias de términos (especificando en todos los campos) indicadas: 1) «eating disorders» AND «childhood trauma» AND «body image», 2) «eating disorders» AND «life stressful events» AND «body image», 3) «eating disorders» AND «childhood trauma» AND «body dissatisfaction», 4) «eating disorders» AND «life stressful events» AND «body dissatisfaction». Se cruzaron también en lugar de «eating disorders», los términos «bulimia nervosa», «anorexia nervosa», «binge eating disorder», y «disordered eating» con sus equivalentes en castellano. Se finalizó la búsqueda el 15 de junio del 2020. También se realizó una búsqueda a través de la bibliografía de los artículos finales. El proceso se indica en el diagrama de flujo a continuación. 


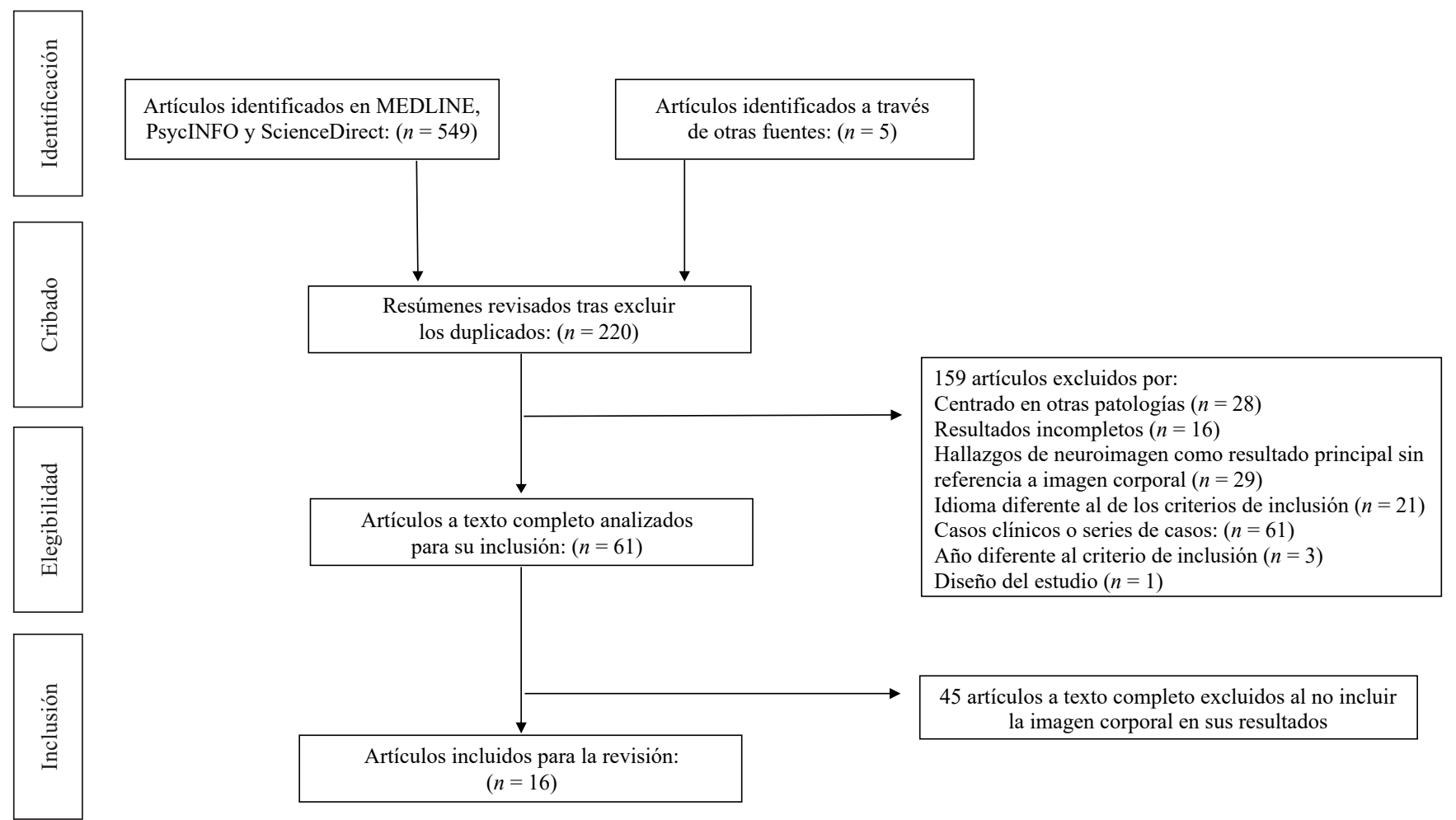

Figura 1. Diagrama de flujo.

\section{Selección y elegibilidad de los estudios}

Cuatro de los seis autores revisaron los resúmenes de los artículos una vez eliminados los duplicados; si había duda en relación a la elegibilidad decidían de forma consensuada los otros dos autores del grupo de trabajo. Los criterios de selección de los estudios fueron: (1) los trabajos trasversales y longitudinales; (2) los que evaluaban la relación entre experiencias adversas vitales a lo largo de infancia, adolescencia y edad adulta, y la clínica alimentaria; (3) los realizados en pacientes con TCA y en población no clínica con presencia de hábitos alimentarios inadecuados; (4) los publicados entre el 2010 y el 2020, en lengua inglesa, francesa, italiana o castellana. En cuanto a los criterios de exclusión fueron los estudios de casos clínicos aislados, los que se centraban en otras patologías, y trabajos con $N$ inferior a 30 .

\section{Proceso de extracción de los datos}

Los datos de los trabajos analizados se han extraído en relación al tipo de estudio, la población en la cual se llevó a cabo, la $n$ muestral, el tipo de experiencia adversa vital recogida, los instrumentos de evaluación utilizados en la metodología, y los resultados relativos a la relación entre experiencia adversa, distorsión e insatisfacción corporal. La extracción fue realizada por dos autores y, cuando no había consenso decidía un tercero del grupo.

\section{Codificación y análisis de los datos}

Las variables se codificaron y registraron en hojas predefinidas por dos de los autores. De cada trabajo se indicó el autor, el año y el país de realización, además de dividirlo según fuesen transversales o longitudinales ya que se permite sacar conclusiones con respecto al tipo de relación causal. Con respecto a los participantes se distinguió entre pacientes con TCA en tratamiento, y población no clínica, cuando los participantes provenían de grupos sin diagnostico establecido, codificando la proveniencia de los mismos como población clínica en el primer caso, y comunitaria en el segundo. De la muestra se registró el sexo (porcentaje de mujeres) para poder valorar diferencia de género y la edad con rango y deviación típica. Las características de las experiencias vitales adversas que se tuvieron en cuenta fueron el tipo específico, si se había repetido en el tiempo, la etapa vital en la cual había ocurrido, el impacto subjetivo de la misma y el número de situaciones adversas vividas. En relación a los cuestionarios empleados en los estudios se han codificados según recogiesen las variables experiencia adversa vital y los usados para la imagen corporal. De los 
resultados sobre la relación entre la experiencia adversa vital y la imagen corporal se han registrado el tipo de correlación y la presencia de eventuales factores mediadores si los autores los describían; se han registrado además diferencias de género. Al no ser el objetivo de este trabajo la realización de un meta-análisis, ni incluir la revisión estudios comparativos de tratamiento, no se calcularon los tamaños de efecto de los diferentes trabajos. Se ha realizado posteriormente un análisis cualitativo y cuantitativo descriptivo que se resume en la Tabla 1.

\section{Evaluación del riesgo de sesgo de los estudios individuales}

Para determinar la calidad de los estudios, dos autores utilizaron de manera independiente la herramienta de evaluación de riesgo de sesgo para estudios no randomizados (por sus siglas en ingles RoBANS) (Kim et al., 2013) dado que trata de estudios observacionales. Esta herramienta incluye seis posibles áreas con riesgo de sesgo: selección de participantes; variables de confusión; medida de exposición; cegamiento de la evaluación de resultado; resultados incompletos; e informe selectivo de resultados. De cada área se indica si el riesgo de sesgo es alto, bajo o no claro. En aquellos casos en los que no hubiera un acuerdo entre los autores en alguna de las áreas evaluadas, el autor principal de este trabajo fue consultado y tomaba la decisión final.

\section{Resultados}

\section{Análisis descriptivo de los estudios}

De los 549 títulos iniciales identificados tras la búsqueda inicial, 220 fueron eliminados al tratarse de duplicados. Los 329 resúmenes restantes fueron analizados y se eligieron 56 artículos para el análisis a texto completo, a los cuales se añadieron 5 artículos provenientes de la revisión de bibliografía. A señalar una revisión no sistemática (Madowitz et al, 2015) de la cual se consideraron elegibles 3 artículos (Treurer et al., 2005; Eubanks et al., 2006; Preti et al., 2006) que finalmente se excluyeron al no cumplir el criterio del año de realización del estudio (2010-2020). Tras eliminar los 45 trabajos que no incluyan la imagen corporal en la presentación de los resultados, finalmente se han incluido en la revisión los $16(26.2 \%)$ que hacían referencia o evaluaban específicamente la relación entre las experiencias adversas vitales y la alteración con la imagen corporal. Los trabajos son representativos de 3 continentes habiéndose realizado en Europa (Villarroel et al., 2012; Guillaume et al., 2016; Tagay, et al., 2014; Backholm et al., 2013; Kothari et al., 2015; Roenholt et al., 2012; Longo et al., 2020), Américas (Mason et al., 2015; Hicks White et al., 2018; Groff Stephen y Wilke, 2016; Dunkley et al., 2010; Bakalar et al., 2018; Richardson et al., 2014; Vartanian et al., 2018) y Oceanía (Brooke y Mussap, 2013; Mitchinson et al., 2018).

En relación a los participantes el $37.5 \%$ se ha realizado en población TCA (Hicks White et al., 2018; Guillaume et al., 2016; Backholm et al., 2013; Dunkley et al., 2010; Tagay et al., 2014, Longo et al., 2020) y el resto $(62.5 \%)$ en población sin diagnóstico clínico (Richardson et al., 2014; Mason et al., 2015; Mitchison et al, 2018; Groff Stephen y Wilke, 2016; Vartanian et al., 2018; Roenholt et al., 2012; Brooke y Mussap, 2012; Villarroel et al., 2012; Bakalar et al., 2018), con la excepción de que en uno parte de la muestra tenía antecedentes de TCA (Kothari et al., 2015). Las $n$ muestrales en los estudios de población clínica son inferiores a los 200 pacientes excepto en el de Backholm et al., (2013) en el cual los participantes se reclutan desde una base de datos nacional sueca, donde están incluidos todos los pacientes que habían sido tratados por TCA, y que llega a los 4524. En los otros trabajos los participantes se reclutan entre pacientes atendidos a nivel ambulatorio u hospitalario (Hicks White et al., 2018; Guillaume et al., 2016; Dunkley et al., 2010; Tagay et al., 2014; Longo et al., 2020). Las categorías diagnosticas evaluadas son anorexia nerviosa $(n=1141)$, bulimia nerviosa $(n=$ $1591)$, trastorno por atracón $(n=531)$ y TCA no especificado $(n=1853)$ y el $\%$ de mujeres representado varía entre el $72 \%$ y el $100 \%$. Las muestras de los estudios con población comunitaria se han reclutado entre jóvenes universitarias (Groff Stephen y Wilke, 2016; Brooke y Mussap, 2013; Villarroel et al., 2012), nacidos en 1984 (Roenholt et al., 2012), adolescentes de institutos (Richardson et al., 2014), hijos de enfermeras (Mason et al., 2015), mujeres embarazadas (Kothari et al., 2015), jóvenes adultos entre 18 y 30 años mediante publicidad en plataforma digital (Vartanian et al., 2018), jóvenes de más de 15 años representativos de barrios rurales y urbanos del sud de Australia (Mitchison et al., 2018) y personal militar (Bakalar et al., 2018) con un porcentaje de mujeres entre el $47 \%$ y el $100 \%$. El estudio de Vartanian et al. (2018) hace referencia a un tercer género que define como «otro», aunque no se indica a que se refiere en concreto. En cuanto a la edad de las muestras en 5 $(31.2 \%)$ trabajos se incluye población infanto-juvenil (Hicks White et al., 2018; Guillaume et al., 2016; Mitchison et al., 2018; Richardson et al., 2014; Mason et al., 2015), siendo el resto en adultos (rango: 18-100).

Respecto al diseño de los estudios 3 (18.7\%) son longitudinales (Richardson et al., 2014; Kothari et al., 2015; 


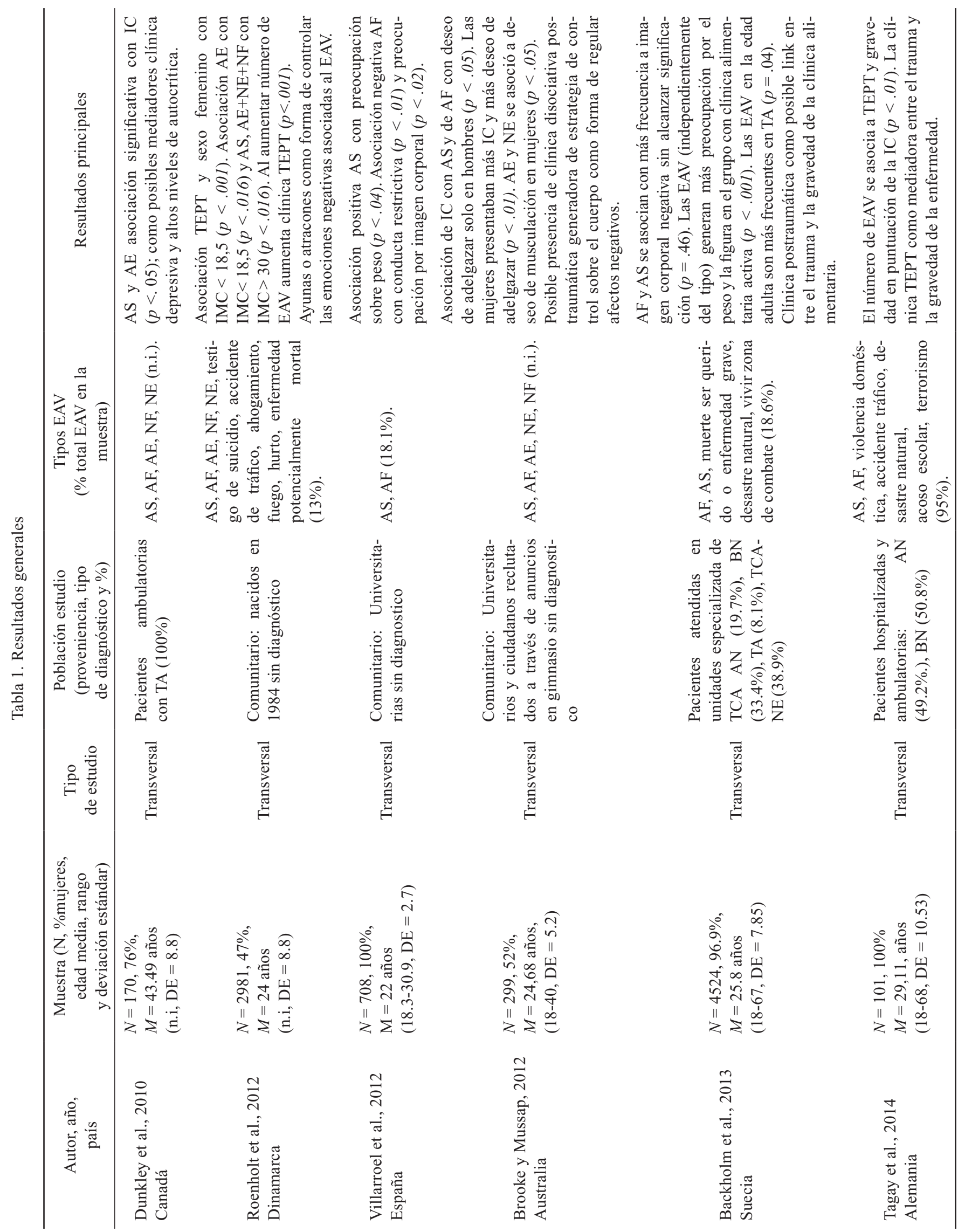




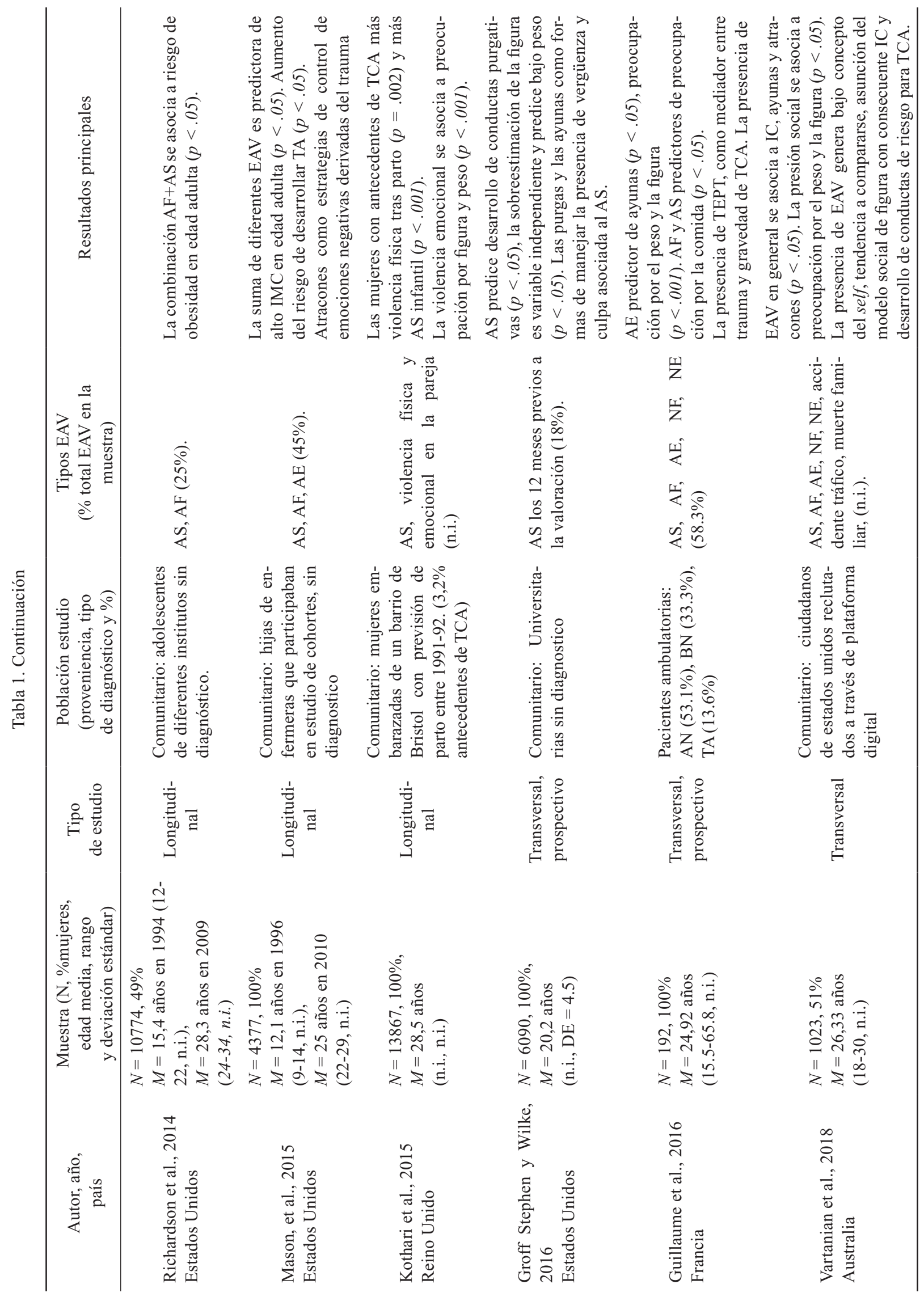




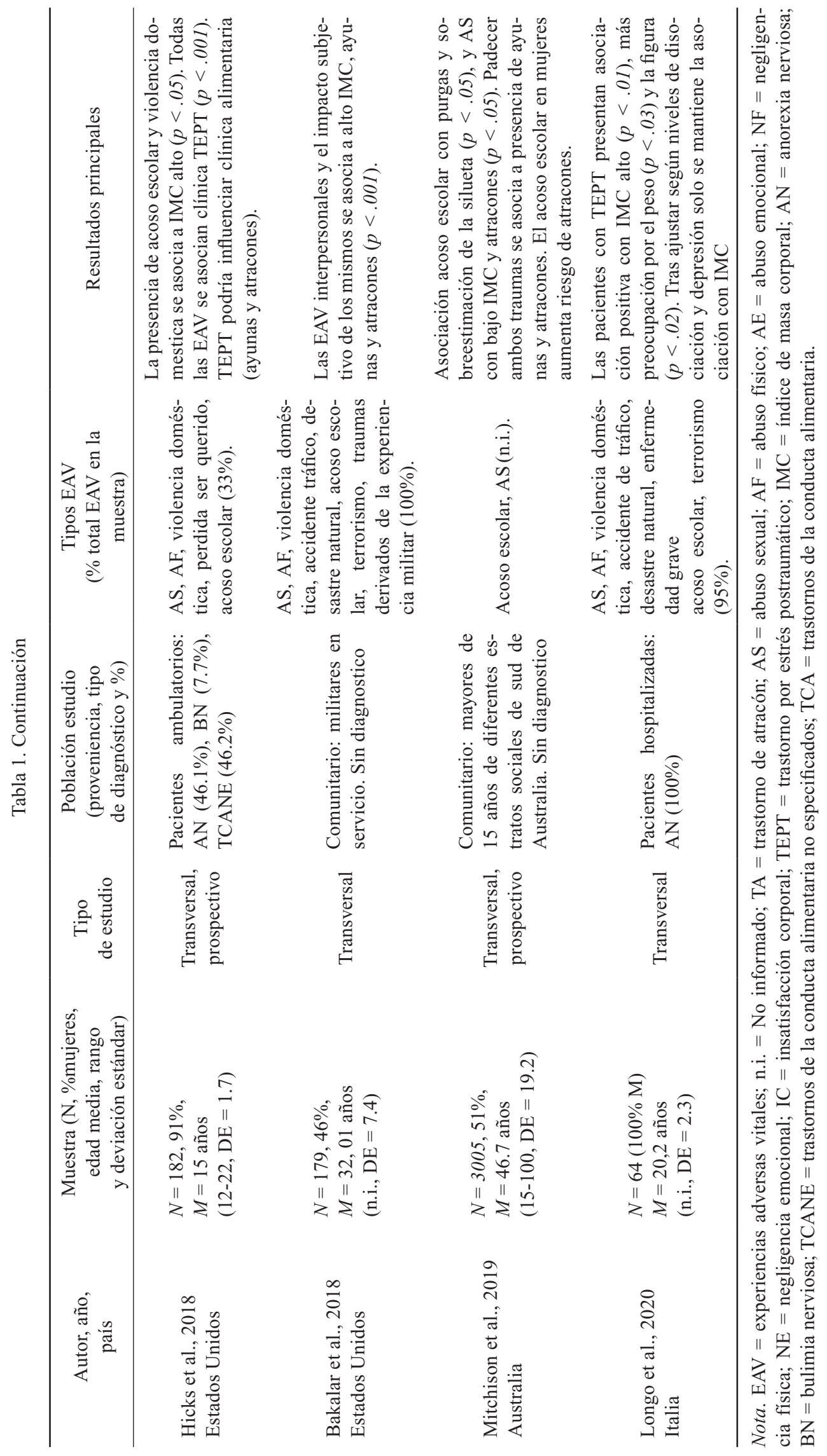


Mason et al., 2015), 4 (25\%) transversales prospectivos (Mitchison et al, 2018; Hicks White et al, 2018; Groff Stephen y Wilke, 2016; Guillaume et al., 2016), 9 $(56.2 \%)$ transversales retrospectivos (Backholm et al., 2013; Dunkley et al., 2010; Vartanian et al., 2018; Tagay et al., 2014; Brooke y Mussap, 2012; Villarroel et al., 2012; Roenholt et al, 2012; Bakalar et al., 2018; Longo et al., 2020).

Dado el aumento de interés sobre la repercusión de las experiencias adversas vitales en la salud mental, en los últimos años se han desarrollado diferentes test validados para la recogida de las mismas, aunque en muchos trabajos se suelen utilizar cuestionarios elaborados por los autores dificultando las generalizaciones (Donald, 2012). De los trabajos revisados (en la Tabla 2 se resumen los principales instrumentos utilizados en los trabajos de esta revisión) en el $43.7 \%$ de los casos se recurre a cuestionarios propios (Backholm et al., 2013; Kothari et al., 2015; Hicks White et al., 2018; Groff Stephens y Wilke, 2016; Richardson et al., 2014; Mason et al., 2015; Roenholt et al., 2012), y en el resto, a no ser que se evalúen situaciones específicas como en el estudio de Bakalar et al, (2018) en personal militar, el test más utilizado es el cuestionario de trauma infantil (CTQ; Bernstein y Fink, 1998). En cuanto a la evaluación de la imagen corporal, en nuestra revisión $4(25 \%)$ estudios la evalúan mediante el IMC (Richardson et al., 2014; Mason et al., 2015; Roenholt et al., 2012; Hicks White et al., 2018), en dos (12.5\%) se ha utilizado entrevista directa preguntando a los participantes cual creían que era su peso y comparándolo con el real para evaluar la distorsión corporal y si estaban satisfechos con el mismo para evaluar la insatisfacción (Groff Stephen y Wilke, 2016; Mitchison et al., 2018). En los otros se han utilizados cuestionarios validados siendo el más usado (56.2\% de los estudios) el cuestionario de evaluación de trastornos alimentarios (EDE-Q; Fairburn y Beglin, 1994), autoadministrado con 37 ítems (respuesta tipo Likert) que contiene 4 subescalas que evalúan las preocupaciones sobre la silueta (Backholm et al, 2013; Bakalar et al., 2018; Brooke y Mussap, 2013; Guillaume et al., 2016; Kothari et al, 2015; Mitchison et al, 2018; Vartanian et al, 2018; Villarroel et al., 2012; Longo et al., 2020), cuya consistencia interna es de 0.92 . También se han utilizado (Brooke y Mussap, 2013; Tagay et al., 2014) las subescalas de obsesión para adelgazar del inventario de trastornos alimentarios versión 2 (EDI-2; Garner, 1993) y 3 (Garner, 2004), cuestionarios de 91 ítems que evalúan diferentes aspectos cognitivos y conductuales de los TCA (consistencia interna: 0.82); la escala sobre obsesión para la musculación (consistencia interna: 0.82) de McCreary (2007) en el estudio de Brooke y Mussap
(2012), y el Body shape questionnaire (BSQ; Cooper et al., 1987), cuestionario auto aplicado de 34 ítems que evalúa miedo a engordar, baja autoestima ligada a la apariencia, insatisfacción corporal y cogniciones asociadas al peso (consistencia interna: 0.95), en el de Dunkley et al., (2010) (véase Tabla 2).

\section{Análisis de la variable experiencias adversas vitales}

Una de las dificultades metodológicas que se encuentran cuando se evalúan las experiencias adversas vitales en TCA es la presencia de diferentes formas de categorizarlas y que, a veces, impiden generalizar los resultados (Trottier, y MacDonald, 2017). Para extraer los datos relativos a las experiencias estudiadas hemos adoptado la clasificación de Kira et al., (2008) ya que permite tener en cuenta dos dimensiones: la que depende del funcionamiento individual y la que se asocia a la experiencia en sí; hemos entonces valorado el tipo específico de situación adversa, la etapa vital en la cual ocurre, el impacto subjetivo de la misma, y el número total en caso de referir más de una (polivictimización) resumiéndose los resultados en la Tabla 3. El 93.7\% de los estudios exploran las experiencias acaecidas durante la infancia, y de estos $5(32.2 \%)$ incluyen la que ocurren en la edad adulta (Bakalar et al., 2018; Mitchinson, et al., 2018; Kothary, et al., 2015; Tagay, et al., 2014; Backolm, et al., 2013). El trabajo de Groff Stephen y Wilke (2016), solo incluye situación adversa en edad adulta (véase Tabla 3 ).

\section{Análisis de los resultados sobre la correlación entre experiencias adversas vitales e imagen corporal}

Si consideramos el tipo específico de situación adversa sea el abuso físico, sea el emocional que el sexual padecido en la infancia se han asociado a un IMC elevado o bajo y a alta insatisfacción corporal; más específicamente, el abuso emocional se reporta con más frecuencia en personas con bajo peso, mientras que el abuso físico y el sexual se relacionan con un peso alto (Mason et al., 2015; Kothary et al. 2015; Dunkley et al., 2010; Richardson et al., 2014; Vartanian et al., 2018; Tagay et al., 2014; Roenholt et al., 2012; Longo et al., 2020). El estudio de Guillaume et al., (2016), en la relación entre experiencias adversas e insatisfacción corporal, distingue como el abuso emocional se asocia con más frecuencia a la preocupación por la figura corporal mientras que el físico se asocia a la preocupación por el peso. En todos los estudios revisados la correlación descrita es positiva excepto en el de Villarroel et al. (2012) que describe una asociación negativa entre abuso físico e insatisfacción corporal. También se ha encontrado asociación positiva entre aco- 


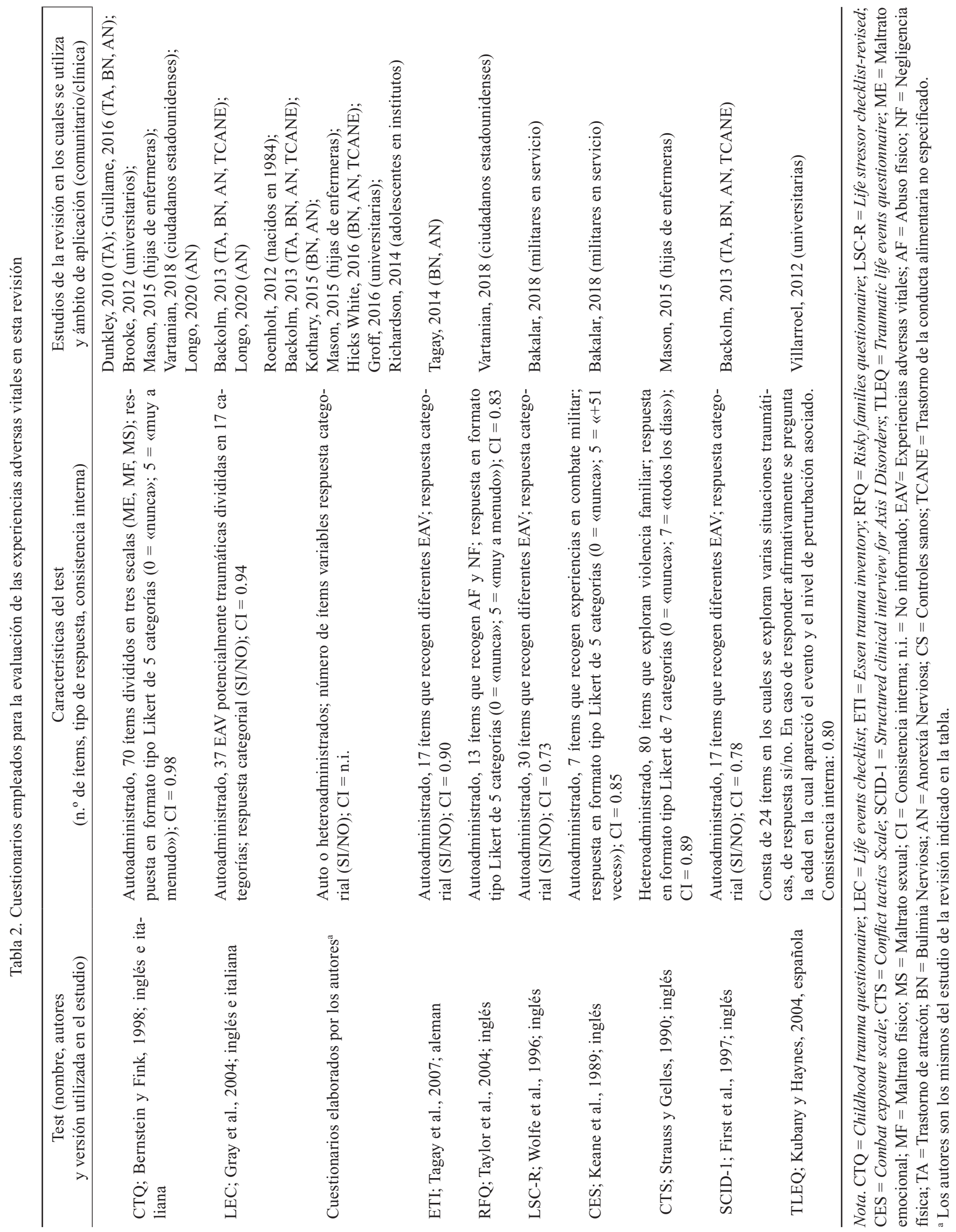


Tabla 3. Características evaluadas de las experiencias adversas vitales

\begin{tabular}{|c|c|c|}
\hline & Experiencia adversa vital & $N$ de estudios $(\%)$ \\
\hline & No realizado por el hombre (ej. desastre natural) & $5(31.25 \%)$ \\
\hline & Abuso Sexual & $16(100 \%)$ \\
\hline & Abuso Físico & $13(81.25 \%)$ \\
\hline & Abuso emocional & $7(43.75 \%)$ \\
\hline & Negligencia emocional & $5(31.25 \%)$ \\
\hline & Negligencia física & $5(31.25 \%)$ \\
\hline & Violencia sexual & $4(25 \%)$ \\
\hline \multirow[t]{9}{*}{ Tipo } & Hurto & $3(18.75 \%)$ \\
\hline & Acoso escolar & $3(18.75 \%)$ \\
\hline & Violencia de genero & $1(6.25 \%)$ \\
\hline & Violencia domestica & $3(18.75 \%)$ \\
\hline & Accidente de trafico & $4(25 \%)$ \\
\hline & Muerte familiar & $4(25 \%)$ \\
\hline & Accidente bélico & $2(12.5 \%)$ \\
\hline & Terrorismo & $3(18.75 \%)$ \\
\hline & Infancia & $10(62.50 \%)$ \\
\hline \multirow[t]{2}{*}{ Etapa en la que ocurrió } & Infancia y edad adulta & $5(31.25 \%)$ \\
\hline & Edad adulta & $1(6.25 \%)$ \\
\hline Polivictimización & $\mathrm{Si}$ & $7(43.75 \%)$ \\
\hline Impacto subjetivo & $\mathrm{Si}$ & $1(6.25 \%)$ \\
\hline
\end{tabular}

so escolar, violencia intrafamiliar e IMC elevado (Mitchison et al., 2018; Hicks White et al., 2018; Longo et al., 2020), violencia en la pareja y aumento de la preocupación entorno a la silueta (Kothari et al., 2015), estrés derivado del combate y riesgo de obesidad (Bakalar et al, 2018). Todos los estudios han evaluado diferentes tipos de situaciones adversas y solo 1 (Groff Stephens y Wilke, 2016) se ha centrado exclusivamente en los efectos de la violencia sexual encontrando asociación positiva con conductas purgativas mientras que la alteración de la imagen corporal parece ser una variable independiente que predice bajo peso. La distorsión de la imagen corporal se ha asociado al acoso escolar en el estudio de Mitchison et al., (2018).

En relación al periodo vital en el cual ocurre la experiencia vital adversa, el $93.3 \%$ de los trabajos exploran la etapa infantil, pero solo en uno se ha introducido la variable edad para estudiar la asociación, describiéndose relación con el IMC alto cuando ocurre antes de los 11 años (Mason et al., 2015). Las experiencias adversas vitales en la etapa adulta se correlacionan positivamente con un IMC alto y se describen más frecuentemente en pacientes con trastorno por atracón o en personas con síntomas bulímicos (Backholm et al., 2013; Kothari et al., 2015; Tagay et al., 2014; Groff Stephens y Wilke, 2016; Mitchison et al., 2018; Bakalar et al., 2018).

La polivictimización se ha evaluado en $7(43.7 \%)$ estudios, sin encontrar asociación con la insatisfacción corporal, ni con la distorsión o el peso; sin embargo, a más experiencias vitales adversas, peor funcionamiento psicosocial y riesgo de presentar trastorno por estrés postraumático y/o sintomatología bulímica (Guillaume et al., 2016; Hicks White et al., 2018; Tagay et al., 2014, Richardson et al., 2018, Mason et al., 2015; Roenholt et al., 2012; Longo et al., 2020).

Finalmente, el impacto subjetivo de la EAV solo es evaluado por Bakalar et al. (2018) quienes reportan correlación positiva con un IMC alto.

Aunque se incluyen ambos géneros en el $62.5 \%$ de los estudios solo en tres (18.7\%) se presentan diferencias en los resultados (Brooke y Mussap, 2012; Mitchison et al., 2018; Roenholt et al., 2012). En general la vivencia de una experiencia potencialmente traumática se asocia a alta insatisfacción corporal sea en hombres que, en mujeres, pero, en los primeros el abuso físico y el sexual se han correlacionado con el deseo de adelga- 
zar, mientras que, en las segundas, el abuso y la negligencia emocional junto a la física determinan el deseo de musculación (Brooke y Mussap, 2012). En mujeres, además se describen relaciones entre polivictimización, estrés postraumático e IMC por debajo de 18 y entre acoso escolar y clínica bulímica (Mitchison et al., 2018). Roenholt et al., (2012) reportan como la presencia de sintomatología postraumática se asocia con bajo IMC en las mujeres y en hombres al elevado.

Con respecto a la presencia de factores mediadores que podrían explicar la relación entre experiencias adversas vitales y la alteración de la imagen corporal, en 6 estudios (37.5\%), se encuentran conductas de restricción y purgativas ligadas al desarrollo de clínica postraumática (Brooke y Mussap, 2012; Backholm et al., 2013; Tagay et al., 2014; Roenholt et al., 2012; Guillaume et al., 2016; Hicks White et al., 2018). La desregulación emocional (Taylor et al., 2004) ha sido reportada en 2 estudios: el longitudinal de Mason et al. (2015) que describe riesgo de desarrollar un trastorno por atracón al encontrar relación significativa entre la polivictimización y la presencia de atracones, y en el de Groff Stephen y Wilke, (2016) que hipotetiza como la culpa y la vergüenza derivadas de una violación pueden llevar a insatisfacción corporal y aumento de peso, también a través del inicio de atracones. Otros mediadores identificados son la clí- nica depresiva, altos niveles de autocrítica y experiencias disociativas (Dunkley et al., 2010; Vartanian et al., 2018; Longo et al., 2020). Por último, señalar el estudio de Bakalar et al. (2018) en el cual se diferencia, al presentar los resultados, entre situaciones adversas interpersonales o no interpersonales: en el primer caso la asociación con IMC elevado es mediada por clínica bulímica, mientras que en el segundo lo es el impacto subjetivo que genera la experiencia. En el 25\% de los estudios no se describen factores mediadores (Villarroel et al., 2012; Richardson et al., 2014; Mitchinson et al., 2018; Kothary et al., 2015).

\section{Análisis de los resultados sobre la evaluación de sesgo de los estudios}

Como se puede observar en la Tabla 4, en el 100\% de los estudios se encontró un riesgo de sesgo bajo en relación al área de informe selectivos de resultados, aunque con respecto al área presentación de resultados, en dos estudios (Richardson et al., 2014, Mason et al., 2015) se detectó un riesgo de sesgo alto al no presentar de forma completa los mismos. En el 37.5\% de los trabajos no se pudo definir el riesgo en relación a la selección de los participantes que se señaló como no claro y en el $62.5 \%$ de los casos había un riesgo de sesgo alto en cuanto al área medidas de exposición.

Tabla 4. Riesgo de sesgo de los estudios incluidos

\begin{tabular}{lcccccc}
\hline \multicolumn{1}{c}{ Estudio } & $\begin{array}{c}\text { Selección } \\
\text { de participantes }\end{array}$ & $\begin{array}{c}\text { Variables } \\
\text { de confusión }\end{array}$ & $\begin{array}{c}\text { Medida } \\
\text { de exposición }\end{array}$ & $\begin{array}{c}\text { Cegamiento } \\
\text { de la evaluación } \\
\text { de resultado }\end{array}$ & $\begin{array}{c}\text { Resultados } \\
\text { incompletos }\end{array}$ & $\begin{array}{c}\text { Informe } \\
\text { selectivo } \\
\text { de resultados }\end{array}$ \\
\hline Backholm et al., 2013 & Bajo & Alto & Bajo & Bajo & Bajo & Bajo \\
Bakalar et al., 2018 & Bajo & Bajo & Alto & Bajo & Bajo & Bajo \\
Brooke y Mussap, 2012 & No claro & Alto & Alto & Bajo & Bajo & Bajo \\
Dunkley et al., 2010 & Bajo & Bajo & Bajo & Bajo & Bajo & Bajo \\
Stephens y Wilke, 2016 & Bajo & Alto & Alto & Bajo & Bajo & Bajo \\
Guillaume et al., 2016 & Bajo & Bajo & Bajo & Bajo & Bajo & Bajo \\
Hicks et al., 2018 & Bajo & Bajo & Bajo & Bajo & Bajo & Bajo \\
Kothari et al., 2015 & Bajo & Bajo & Alto & No claro & Bajo & Bajo \\
Longo et al., 2020 & Bajo & Bajo & Bajo & Bajo & Bajo & Bajo \\
Mason et al., 2015 & No claro & Bajo & Alto & Bajo & Alto & Bajo \\
Mitchison et al., 2019 & Bajo & Alto & Alto & Bajo & Bajo & Bajo \\
Richardson et al., 2014 & No claro & Bajo & Alto & Bajo & Alto & Bajo \\
Roenholt et al., 2012 & No claro & Alto & Bajo & Bajo & Bajo & Bajo \\
Tagay et al., 2014 & No claro & Alto & Alto & Bajo & Bajo & Bajo \\
Vartanian et al., 2018 & Alto & Alto & Alto & Bajo & Bajo & Bajo \\
Villarroel et al., 2012 & No claro & Bajo & Alto & Bajo & Bajo & Bajo \\
\hline
\end{tabular}




\section{Discusión}

El objetivo de este trabajo era revisar y esclarecer la relación entre las experiencias adversas vitales y la imagen corporal en TCA. Resulta pertinente, considerado el aumento de incidencia de estos trastornos, su alta morbimortalidad y su resistencia al tratamiento entre cuyos factores se encuentra la alteración de la imagen corporal, además del aumento del interés del papel de los eventos estresantes en TCA en la última década. A pesar de este incremento muy pocos estudios se centran en la imagen corporal y, hasta donde alcanza nuestro conocimiento, no hay ninguna revisión reciente sobre el tema.

Si bien la mayoría de los trabajos revisados son de los últimos 6 años, (el 68.7\% entre el 2014 y el 2020), solo 4 se han realizado en población clínica TCA, dificultándose la generalización de resultados a este grupo poblacional, siendo el resto de trabajos llevados a cabo en poblaciones en la cuales se identifican hábitos alimentarios inadecuados. Esto se podría explicar siguiendo la idea expuesta por Treasure y colaboradores (Treasure, et al., 2020) según la cual la investigación en TCA es limitada por la escasa financiación viéndose favorecidos otros trastornos como los afectivos.

Con respecto al tipo de estudios realizados, en nuestra revisión prevalecen los estudios transversales y retrospectivos respecto a los longitudinales, siendo aconsejable su aumento, para poder dilucidar el tipo de relación de las diferentes experiencias adversas vitales como se ha señalado ya en anteriores actualizaciones sobre el tema (Trottier y MacDonald, 2017). Por otro lado, tal y como indican algunos autores, la naturaleza de las variables estudiadas podrían limitar la posibilidad de realizar estudios longitudinales prospectivos, siendo la mayoría de los trabajos realizados retrospectivos (Trottier y MacDonald, 2017).

En relación a la población, la más abordada es la comunitaria, encontrándose diferentes tipos de muestras, ayudando a tener una perspectiva colectiva que permite alcanzar a distintos grupos etarios. En el caso de los estudios en población clínica vemos que sigue habiendo un porcentaje elevado de TCA no especificado, a expensas del trastorno por atracón, destacando que todavía se utilizan criterios DSM-4 (American Psychiatric Association, 2002). Considerado el aumento de incidencia de los pacientes con trastorno por atracón sería aconsejable generar nuevos trabajos para poder implementar los nuevos criterios diagnósticos y poder generalizar los resultados a la realidad clínica actual. Un aspecto de considerable relevancia es que únicamente se ha encontrado un trabajo de investigación que contase con un grupo control de comparación, haciéndose patente una de las principales limitaciones en este campo de investigación. Por otro lado, dado que la insatisfacción corporal y la distorsión de la imagen no son específicos de los TCA (De Vignemont, 2009), habiéndose descrito también en otros grupos diagnósticos y en población sin clínica (Sepúlveda, et al., 2004; González-Carrascosa et al., 2013; Rabito-Alcón y Rodríguez-Molina, 2016) sería interesante, a nivel metodológico, introducirlos en futuros trabajos.

En relación al tipo de situación adversa estudiada, en nuestra revisión se han recogido las mismas que se han descrito en diferentes trabajos previos (Lejonklou et al., 2014; Afifi et al., 2017), predominando el abuso físico y el sexual, siendo también relevantes los resultados sobre el abuso emocional. Este último se ha asociado en trabajos anteriores a experiencias disociativas y desregulación emocional (Moulton et al., 2015), así como a alta insatisfacción corporal en población comunitaria y en pacientes con trastorno por atracón (Kenardy y Ball, 1998; Grilo y Masheb, 2001; Treurer et al., 2005). Algún autor ha señalado como el acoso escolar podría ser un factor de riesgo para el desarrollo del trastorno por atracón, donde se suelen encontrar altos niveles de insatisfacción corporal (Salwen et al., 2015). En nuestra revisión tanto el abuso emocional como el acoso escolar parecen estar relacionados al aspecto cognitivo de la insatisfacción corporal y a baja autoestima. Es probable que las emociones asociadas a dichas experiencias se podrían «transformar» en cogniciones negativas o excesiva preocupación por el cuerpo en personas con rasgos obsesivos y baja autoestima características que se han encontrado en todos los pacientes con TCA (Treasure et al., 2020).

Al considerar los resultados relativos al tipo de instrumentos utilizados llama la atención como casi la mitad de los estudios revisados utiliza todavía listados de cuestionarios propios para recoger las experiencias potencialmente traumáticas en lugar de utilizar cuestionarios validados, exceptuando la evaluación de experiencias en la infancia que casi siempre se miden con el cuestionario de trauma infantil (Bernstein y Fink, 1998). Teniendo en cuenta que en los últimos años ha habido un aumento de la elaboración de test específicos por el aumento de interés del estudio de experiencias traumáticas en salud mental (Donald, 2012) sería aconsejable utilizar instrumentos reconocidos para facilitar la replicabilidad de los trabajos y la generalización de los resultados. En cuanto a la evaluación de la distorsión de la imagen corporal se encuentra un problema similar al utilizar, como medida de evaluación, el IMC auto reportado por los participantes que se compara con el real. Esto podría representar un sesgo ya que no es una medida objetiva y 
habiendo en la actualidad técnicas de estimación corporal mediante la realidad virtual que han demostrado su mayor precisión y podrían considerarse como una alternativa fiable (Marco et al., 2004). Otro problema podría derivar de que todavía no hay consenso sobre la alteración de la imagen corporal en TCA (Sepúlveda et al., 2004). Autores han recordado que en la estimación de la figura corporal puede haber una proyección de actitudes y emociones negativas sobre uno mismo desplazadas hacia el cuerpo y creen que la evaluación de la figura corporal debería de ser sustituida por la evaluación actitudinal, al no considerar que haya una alteración perceptiva corporal en los TCA sino una valoración negativa del cuerpo (Hsu y Sobkiewicz, 1991). Evaluar solo la IC con todos sus aspectos permitiría entonces homogeneizar los resultados de los diferentes estudios ya que se dispone de test validados en diferentes poblaciones.

Con respecto al tipo de relación entre experiencias adversas vitales y la insatisfacción corporal, tan solo tres de los trabajos revisados profundizan sobre los aspectos cognitivos de la misma identificando excesiva autocritica, deseo de musculación y bajo concepto de uno mismo en contexto de diferentes situaciones traumáticas. Se podría pensar que la experiencia vivida deja un sentimiento de inseguridad o indefensión a los cuales la persona se sobrepone con mecanismos de control sobre su cuerpo para tener una falsa sensación de seguridad como señalado por Thompson, y Wonderlich (2004). Aunque todavía no hay datos suficientes para considerarlo factores pronósticos de la enfermedad (Caslini et al, 2016), lo que parece más claro en otros trabajos sobre TCA y experiencias traumáticas es la presencia de factores mediadores entre dichas situaciones y la psicopatología alimentaria representados por la clínica postraumática (Vierling et al., 2015), la disociativa (Moulton et al., 2015; Palmisano et al., 2018), y la desregulación emocional (Demirci, 2018; Malinauskiene y Malinauska, 2018). Se cree que la exposición a experiencias adversas vitales podría inducir conductas alimentarias anómalas como los atracones o la conducta restrictiva en personas con dificultad para reconocer y manejar las experiencias negativas generadas por dichas experiencias (Brustenghi et al., 2019; Fox y Power, 2009). Estos mediadores podrían ser dianas de tratamiento específicas también en el abordaje de la alteración de la imagen corporal presente en los TCA. Se sugiere que los síntomas postraumáticos se asocian a insatisfacción corporal en pacientes con anorexia nerviosa restrictiva que han padecido abuso sexual (Madowitz et al., 2015). De los mediadores reportados en nuestra revisión parece que la clínica postraumática influencia sea los factores cognitivos asociados a la insatisfacción corporal, sea las conductas alimentarias asociadas a aumento o disminución del IMC. La desregulación emocional sería el factor que facilitaría el desarrollo de atracones cuando se intentan manejar emociones como culpa y la vergüenza tras un abuso sexual o el malestar derivado de la suma de diferentes experiencias adversas. Dicha desregulación se ha identificado en los diferentes subtipos de TCA asociándose, en otros estudios, a subgrupos de pacientes con preocupación por el peso y la figura, además de encontrarse asociada a más conductas impulsivas sobre todo en pacientes con trastorno por atracón (Monell et al., 2018). La impulsividad y el perfeccionismo son dos dimensiones identificadas en los TCA representando los extremos de una misma dimensión fenomenológica del espectro obsesivo-compulsivo (Treasure et al., 2020). En alguna revisión se ha sugerido que el perfeccionismo podría ser un factor predictivo de la evolución de los TCA (Egan et al., 2013) y se ha señalado como una autorrepresentación de «self» perfeccionista se ha asocia a más vulnerabilidad en las relaciones y riesgo de desarrollo de alteraciones en la alimentación (Chemisquy, y Helguera, 2018). Se podrían imaginar los atracones y las conductas restrictivas los epifenómenos de estas dos dimensiones. En los artículos revisados la asociación con la insatisfacción corporal o el peso siempre se encuentra mediada a través de la desregulación emocional por el desarrollo de atracones o ayunas, como forma de controlar el cuerpo; se podría hipotetizar que dicha desregulación se relaciona con la insatisfacción corporal a través de la impulsividad y del perfeccionismo (Donahue et al., 2018).

Finalmente, con respecto a las diferencias entre géneros no es posible sacar conclusiones ya que de los tres trabajos que las describen, solo uno reporta una asociación con la insatisfacción corporal diferente en hombres y mujeres dependiendo de si se ha padecido abuso sexual en los primeros y emocional en las segundas. Aunque los TCA se presentan predominantemente en mujeres sería interesante poder incrementar, a la hora de replicar estudios, la presencia de sexo masculino dado que hay un aumento de otras presentaciones fenomenológicas, todavía no incluidas como categorías diagnosticas, como la vigorexia, donde la imagen corporal cobra protagonismo (Villarino Marín, 2012). Además, esclarecer diferencias de género permitiría un abordaje más específico con los pacientes.

En cuanto a las limitaciones a las que se ha visto sujeto este trabajo de investigación, hay que destacar el escaso número de estudios que se han podido incluir sobre todo los realizados en población clínica, y dentro de estos, la ausencia de trabajos longitudinales que permitan sacar conclusiones sobre la relación entre experiencias adversas vitales e imagen corporal. Por otro lado, el 
tiempo de inclusión de la búsqueda para considerar elegibles los artículos es relativamente breve, y se han excluido estudios con muestra inferiores a 30 . Además, los estudios incluidos presentan un riesgo de sesgo elevado en relación a la medida de exposición representada por las experiencias adversas vitales indicando un problema de heterogeneidad cuando se intenta inferir conclusiones en los estudios sobre estas variables.

En conclusión, hay pocos estudios sobre experiencias adversas vitales, TCA e imagen corporal $(37.5 \%$ en nuestra revisión), y ninguno longitudinal. El abuso sexual, el físico, la violencia intrafamiliar, las experiencias de combate, el acoso escolar y las experiencias adversas interpersonales identifican subgrupo de personas con sobrepeso y alta insatisfacción corporal. Se están considerando para el análisis de la relación diferentes factores, a parte de la experiencia específica, como el periodo en que ocurrió, el impacto subjetivo que ha tenido, si ha sido puntual o se ha repetido, la polivictimización. La repetición de experiencias traumáticas se ha asociado al desarrollo de trastorno por estrés postraumático, y este a la presencia de clínica bulímica. La relación con la imagen corporal es mediada por clínica postraumática y la desregulación emocional. Parece que los resultados de los estudios revisados en general refuerzan la hipótesis de la clínica alimentaria como una respuesta a un mal manejo de emociones negativas derivadas de diferentes experiencias adversas vitales y que este mecanismo podría estar también a la base de la insatisfacción corporal de los pacientes. Falta esclarecer su relación con la distorsión de la imagen corporal. Falta profundizar en nuevos abordajes que permitan incidir en el tratamiento de los factores mediadores: la clínica postraumática y la desregulación emocional, según los resultados de nuestra revisión, podrían ser unas nuevas dianas de tratamiento y, aun considerando la escasez de estudios en población clínica, la muestra TCA aquí representada es de 9640 pacientes. Para elaborar nuevos protocolos terapéuticos, se podrían también considerar los diferentes estudios que evalúan la relación entre TCA, vinculo y desregulación emocional (Burns et al., 2012; Richter et al., 2009). Son los padres, interaccionando con sus hijos en el juego, tocándolos, calmándolos en sus emociones, y ayudándoles a reconocer cuando están mal, quienes inician la construcción de su identidad e imagen corporal (Troisi et al., 2006). A modo de ejemplo, aunque todavía no hay respaldo porqué los datos son inconsistentes, nuevos abordajes podrían venir de las terapias de tercera generación (Hepworth, 2011). En unas patologías que representan un alto coste para la sociedad y un gran sufrimiento para las pacientes y sus allegados no habría que cerrar ninguna puerta.

\section{Conflictos de intereses}

Los autores declaran no tener conflictos de intereses.

\section{Referencias}

Afifi, T.O., Sareen, J., Fortier, J., Taillieu, T., Turner, S., Cheung, K., y Henriksen, C.A. (2017). Child maltreatment and eating disorders among men and women in adulthood: Results from a nationally representative United States sample. International Journal of Eating Disorders, 50, 1281-1296. https://doi. org/10.1002/eat.22783

American Psychiatric Association. (2014). DSM-5. Guía de consulta de los criterios diagnósticos del DSM-5. Editorial Médica Panamericana S.A.

American Psychiatric Association. (2002). Manual Diagnóstico y Estadístico de los Trastornos Mentales DSM-IV-TR. Massón.

Anderluh, M.B., Tchanturia, K., Rabe-Hesketh, S., y Treasure, J. (2003). Childhood obsessive-compulsive personality traits in adult women with eating disorders: defining a broader eating disorder phenotype. American Journal of Psychiatry, 160(2), 242-247. https://doi.org/10.1176/appi.ajp.160.2.242

* Backholm, K., Isomaa, R. y Birgegard, A. (2013). The prevalence and impact of trauma history in eating disorder patients. European Journal of psychotraumatology, 4, 22482-22489. https://doi.org/10.3402/ejpt.v4i0.22482

* Bakalar, J.L., Barmine, M., Druskin, L., Olsen, C.H., Quinlan, J., Sbrocco T., y Tanofsky-Kraff, M. (2018). Childhood adverse life events disordered eating, and body mass index in US Military service members. International Journal of Eating Disorders, 51, 465-469. https://doi.org/10.1002/eat.22851

Bedoya Hernández, M.H. y Marín Cortés. A.F. (2010). Cuerpo vivido e identidad narrativa en mujeres diagnosticadas con trastornos de la conducta alimentaria. Revista Colombiana de Psiquiatría, 39 (3), 544-555. http://www.scielo.org.co/pdf/ $\mathrm{rcp} / \mathrm{v} 39 \mathrm{n} 3 / \mathrm{v} 39 \mathrm{n} 3 \mathrm{a} 08 . \mathrm{pdf}$

Bernstein, D.P. y Fink, L. (1998). Childhood Trauma Questionnaire manual. The psychological corporation.

* Brooke, L. y Mussap, A.J. (2013). Brief report: Maltreatment in childhood and body concerns in adulthood. Journal of Health Psychology 18 (5), 620-626. https://doi.org/10.1177/ 1359105312454036.

Brustenghi, F., Mezzetti F.A., Di Sarno, C. Giulietti, C., Moretti, P., y Tortorella, A. (2019). Eating disorders: the role of childhood trauma and the emotion regulation. Psychiatria Danubina, 31 (3), 509-511. https://pubmed.ncbi.nlm.nih. gov/31488781/

Burns, E.E., Fischer, S., Jackson, J.L, y Harding, H.J. (2012). Deficits in emotion regulation mediate the relationship between childhood abuse and later eating disorder symptoms. Child Abuse y Neglect, 36 (1), 32-39. https://doi.org/10.1016/j. chiabu.2011.08.005

Carretero-Garcia, A. Sánchez Planell, L., Doval, E., Rusiñol Estragués, J., Raich Escursell, R.M., y Vanderlinden, J. (2012). Repeated traumatic experiences in eating disorders and their association with eating symptoms. Eating and Weight Disorders, 17 (4), e267-e273. https://doi.org/10.1007/ BF03325137 
Caslini, M., Bartoli, F., Crocamo, C., Dakanalis, A., Clerici, M., y Carrá, G. (2016). Disentangling the association between child abuse and eating disorders: a systematic review and metaanalysis. Psychosomatic Medicine, 78 (1), 79-90. http://doi. org/ 10.1097/PSY.0000000000000233

Castejón Martínez, M.A., Berengüí Gil, R., y Garcés de los Fayos Ruiz, E. (2016). Relación del índice de masa corporal, percepción de peso y variables relacionadas con los trastornos de la conducta alimentaria en estudiantes universitarios. Nutrición clínica y dietética hospitalaria, 36 (1), 54-63 doi: 10.12873/361castejon. https://revista.nutricion.org/PDF/361 castejon.pdf

Chemisquy, S., y Helguera, G. P. (2018). Impacto de la autopresentación perfeccionista en la salud infantil y adolescente: Una revisión sistemática. Revista de Psicopatología y Psicología Clínica, 23(2), 149-161. https://doi.org/10.5944/ rppc.vol.23.num.2.2018.20095

Cook-Cottone, C.P. (2015). Incorporating positive body image into the treatment of eating disorders: A model for attunement and mindful self-care. Body Image, 14, 158-167. https://doi. org/10.1016/j.bodyim.2015.03.004

Cooper, P.J., Taylor, M.J., Cooper, Z., y Fairburn, C.G. (1987). The development and validation of the body shape questionnaire. Journal of Eating Disorders, 6, 485-494. https://doi. org/10.1002/1098-108X(198707)6:4<485::AIDEAT2260060405>3.0.CO;2-O

Demirci, E. (2018). Non suicidal self-injury, emotional eating and insomnia after child sexual abuse: Are those symptoms related to emotion regulation? Journal of Forensic and Legal Medicine, 53, 17-21. https://doi.org/10.1016/j.jflm.2017.10.012

Devi, F., Shahwan, S., Lin Teh, W., Sambasivam, R., Jue Zhang, Y., Wen Lau, Y., How Ong, S., Fung, D., Gupta, B., Chong, S.A., Subramaniam, M. (2019). The prevalence of childhood trauma in psychiatric outpatients. Annals General of Psychiatry, 18, 15-22. https://doi.org/10.1186/s12991-0190239-1

De Vignemont F. (2009). Body schema and body image-pros and cons. Neuropsychologia, 48 (3), 669-680. https://doi. org/10.1016/j.neuropsychologia.2009.09.022

* Dunkley, D.M., Masheb, R.M. y Grilo, C.M. (2010). Childhood Maltreatment, Depressive Symptoms, and Body Dissatisfaction in Patients with Binge Eating Disorder: The Mediating Role of Self-Criticism. International Journal of Eating Disorders, 43 (3), 274-281. https://doi.org/10.1002/eat.20796

Donald, J.L. (2012). A review of childhood abuse questionnaires and suggested treatment approaches. En E. S. Kalfolu (Ed.), Sexual Abuse-Breaking the Silence (pp 3-20). In Tech. https:// cdn.intechopen.com/pdfs/33654/InTech-A_review_of_ childhood_abuse_questionnaires_and_suggested_treatment_ approaches.pdf

Donahue, J.M., Reilly, E.E., Anderson, L.M., Scharmer, C., y Anderson, D.A. (2018). Evaluating Associations Between Perfectionism, Emotion Regulation, and Eating Disorder Symptoms in a Mixed-Gender Sample. Journal of Nervous Mental Disease, 206 (11), 900-904. https://doi.org/10.1097/ NMD.0000000000000895

Egan, S.J., Wade, T.D., y Shafran, R. (2012). El proceso transdiagnóstico del perfeccionismo. Revista de Psicopatología y Psicología Clínica, 17(3), 279-294. https://doi.org/10.5944/ rppc.vol.17.num.3.2012.11844
Eubanks J.R., Kenkel M.Y., y Gardner R.M. (2006). Body-size perception, body-esteem, and parenting history in college women reporting a history of child abuse. Perceptual and Motor Skills, 102 (2):485-497. https://doi.org/10.2466/ pms.102.2.485-497

Fairburn, C.G. y Beglin, S.J. (1994). Assessment of eating disorders: interview or self-report questionnaire? International Journal of Eating Disorders, 16 (4), 363-370. https://doi. org/10.1002/1098-108X(199412)16:4<363:AIDAT2260160405>3.0.CO;2-\#.

Fairburn, C,G, Cooper, Z, y Shafran, R. (2003). Cognitive behaviour therapy for eating disorders: a «transdiagnostic» theory and treatment. Behaviour Research and Therapy, 41 (5), 509-528. https://doi.org/10.1016/s0005-7967(02)00088-8

First, M., Spitzer, R., Gibbon, M., y Williams, J. (1997). Structured Clinical Interview for DSM-IV Axis I Disorders, Research Version. Biometrics Research, New York State Psychiatric Institute, Patient Edition.

Fosse, G.K. y Holen A. (2006). Childhood maltreatment in adult female psychiatric outpatients with eating disorders. Eating Behaviors, 7, 404-409. https://doi.org/10.1016/j.eatbeh.2005.12.006

Fox, J.R.E. y Power M.J. (2009). Eating disorders and multi-level models of emotions: an integrated model. Clinical Psychology Psychotherapy, 16, 240-267. https://doi.org/10.1002/cpp.626

Rabito-Alcón, M.F.R. y Rodríguez-Molina, J.M. (2016). Body dissatisfaction differences and similarities among people with eating disorders, people with gender dysphoria and university students. Revista de Psicopatología y Psicología Clínica, 21(2), 97-104. https://doi.org/10.5944/rppc.vol.21.num.2.2016.16991

Garner, D.M. (1993). Eating disorder inventory-2 professional manual. Psychological Assessment Resources.

Garner, D.M. (2004). Eating disorder inventory-3 professional manual. Odessa: Psychological Assessment Resources.

González-Carrascosa, R., García-Segovia, P., y Martínez-Monzó, J. (2013). Valoración de la imagen corporal y de los comportamientos alimentarios en universitarios. Revista de Psicopatología y Psicología Clínica, 18(1), 45-59. https://doi. org/10.5944/rppc.vol.18.num.1.2013.12762

Gray, M. J., Litz, B.T., Hsu, J.L., y Lombado, T.W. (2004). Psychometric properties of the life events checklist. Assessment, 11 (4), 330-341. https://doi.org/10.1177/1073191104269954

Grilo, M.C. y Masheb, R. M. (2001). Childhood psychological, physical and sexual maltreatment in outpatients with binge eating disorder: frequency and associations with gender, obesity and eating-related psychopathology. Obesity Research,9(5), 320-325. https://doi.org/10.1038/oby.2001.40.

* Groff Stephen, S. y Wilke, D.J. (2016). Sexual violence, weight perception, and eating disorders indicators in college females. Journal of American College Health, 64 (1), 38-47. https:// doi.org/10.1080/07448481.2015.1074237

Grunwald, M., Ettrich, C., Busse, F., Assmann, B., Dähne, A., y Gertz, H.J. (2002). Angle paradigm: A new method to measure right parietal dysfunctions in anorexia nervosa. Archives of Clinical Neuropsychology, 17 (5), 485-96. https://doi. org/10.1016/S0887-6177(01)00131-7

* Guillaume, S. Jaussent, L., Maimoun, L., Ryst, A., Seneque, M., Villain, L., Hamroun, D., Lefebvre, V., Renard, E., y Courtet, $\mathrm{Ph}$. (2016). Associations between adverse childhood experiences and clinical characteristics of eating disorders. 
Scientific Reports, 6, 35761-35767. https://doi.org/10.1038/ srep35761

Hayaki, J., Friedman, M.A., y Brownell, K.D. (2002), Emotional expression and body dissatisfaction. International Journal of Eating Disorders, 31, 57-62. https://doi.org/10.1002/eat.1111

Helverskov, J. L., Clausen, L., Mors, O., Frydenberg, M., Thomsen, P. H., y Rokkedal, K. (2010). Trans-diagnostic outcome of eating disorders: a 30-month follow-up study of 629 patients. European Eating Disorders Review, 18 (6), 453-463. https:// doi.org/10.1002/erv.1025.

Hepworth, N.S. (2011). A mindful eating group as an adjunct to individual treatment for eating disorders: A pilot study. Eating Disorders, 19, 6-16. https://doi.org/10.1080/10640266.2011.5 33601

Herbert, J. (1997). Stress, the brain, and mental illness. British Medical Journal, 315, 530-535. https://doi.org/10.1136/bmj.315.7107.530

* Hicks White, A. A., Pratt, K. J. y Cottrill, C. (2018). The relationship between trauma and weight status among adolescents in eating disorder treatment. Appetite, 129, 62-69. https://doi.org/10.1016/j.appet.2018.06.034.

Hsu, L. G., y Sobkiewicz, T. A. (1991). Body image disturbance: Time to abandon the concept for eating disorders? International Journal of Eating Disorders, 10 (1), 15-30. https://doi. org/10.1002/1098-108X(199101)10:1<15::AIDEAT2260100103>3.0.CO;2-I

Isomaa, R., Backholm, K. y Birgegard, A. (2015). Posttraumatic stress disorder in eating disorder patients: the role of psychological distress and timing of trauma. Psychiatry Research, 230, 506510. https://doi.org/10.1016/j.psychres.2015.09.044

Keane, T.M., Fairbank, J.A., Caddell, J.M., Zimering, R.T., Taylor, K.L., y Mora, C.A. (1989). Clinical evaluation of a measure to assess combat exposure. Psychological Assessment: A Journal of Consulting and Clinical Psychology, 1 (1), 53-55. https:// doi.org/10.1037/1040-3590.1.1.53

Keel, P.K., Dorer, D.J., Franko, D.L., Jackson, S.C., y Herzog, D.B. (2005). Post remission predictors of relapse in women with eating disorders. American Journal of Psychiatry, 162 (12), 2263-2268. https://doi.org/10.1176/appi.ajp.162.12.2263

Kenardy, J. y Ball, K. (1998). Disordered eating, weight dissatisfaction and dieting in relation to unwanted childhood sexual experiences in a community sample. Journal of Psychosomatics Research, 44,327-337. https://doi.org/10.1016/ s0022-3999(97)00258-4

Kim, S.Y., Park, J. E., Lee, Y. J., Seo, H. J., Sheen, S. S., Hahn, S., Jang, B. H., y Son, H. J. (2013). Testing a tool for assessing the risk of bias for non-randomized studies showed moderate reliability and promising validity. Journal of Clinical Epidemiology, 66, 408-414. https://doi.org/10.1016/j. jclinepi.2012.09.016.

Kira, I.A., Lewandowski, L., Templin, T., Ramaswamy, V., Ozkan, B., y Mohanesh, J. (2008). Measuring cumulative trauma dose, types, and profiles using a development-based taxonomy of traumas. Traumatology, 14 (2), 62-87. https://doi. org/10.1177/1534765608319324

* Kothari, R., Easter, A., Lewis, R., Howard, L.M., y Micali, N. (2015). Intimate Partner Violence among Women with Eating Disorders during the Perinatal Period. International Journal of Eating Disorders, 48, 727-735. https://doi.org/10.1002/ eat. 22429
Kubany, E. S. y Haynes, S. N. (2004). Traumatic life events Questionnaire. Western Psychological Services.

Lasegue, C. H. (1873). De l'anorexie hysterique. Extraido de Archives generales de Medicine, 21 (1), 386-403.

Lejonklou, A., Nilsson, D., y Holmqvist, R. (2014). Variants of potentially traumatizing life events in eating disorders patients. Psychological Trauma, 6 (6), 661-667. https://doi. org/10.1155/2018/9814358

Lethbridge, J., Watson, H.J., Egan, S.J., Street, H., y Nathan, P.R. (2011). The role of perfectionism, dichotomous thinking, shape and weight overvaluation, and conditional goal setting in eating disorders. Eating Behaviors, 12 (3), 200-206. https:// doi.org/ 10.1016/j.eatbeh.2011.04.003. PMID: 21741018

* Longo, P., Marzola, E., De Bacco, C., Demarchi, M., y AbbateDaga, G. (2020). Young patientes with anorexia nerviosa: The contribution of post-traumatic stress disorder and traumatic events. Medicina, 57 (1), 2. http://dx.doi.org/10.3390/ medicina57010002

Madowitz, J., Matheson, B.E. y Liang, G. (2015). The relationship between eating disorders and sexual trauma. Eating and weight disorders, 20 (3), 281-293. https://doi.org/10.1007/ s40519-015-0195-y

Malinauskiene, V. y Malinauska, R. (2018). Lifetime Traumatic Experiences and Disordered Eating among University Students: The Role of Posttraumatic Stress Symptoms. BioMed Research International, Jan 18, 9814358. https://doi. org/10.1155/2018/9814358.

Marco H., Botella C. y Perpiña C. (2004). Tratamiento de las alteraciones de la imagen corporal en los trastornos de la conducta alimentaria. Académica Española.

* Mason, S. M., MacLehose, R. F., y Katz-Wise, S. L., Austin, S. B., Neumark-Sztainer, D., Harlow, B. L. y Rich-Edwards, J. W. (2015). Childhood abuse victimization, stress-related eating and weight status in young women. Annals Epidemiology, 25 (10), 760-766. https://doi.org/10.1016/j. annepidem.2015.06.081

McCreary, D. R. (2007). The Drive for Muscularity Scale: Description, psychometrics, and research findings. In J.K. Thompson y G. Cafri (Ed.), The muscular ideal: Psychological, social, and medical perspectives (pp. 87-106). American Psychological Association. https://doi.org/10.1037/11581-004

Micali, N., Martini, M. G., Thomas, J. J. Eddy, K. T., Kothari, R., Russell, E., Bulik, C. M., y Treasure, J. (2017). Lifetime and 12-month prevalence of eating disorders amongst women in mid-life: a population-based study of diagnoses and risk factors. BMC Medicine 15, 12. https://doi.org/10.1186/ s12916-016-0766-4

Mills, P., Newman, E. F., Cossar, J., y Murray, G. (2015). Emotional maltreatment and disordered eating in adolescents: testing the mediating role of emotion regulation. Child Abuse y neglect, 39, 156-166. https://doi.org/10.1016/j.chiabu.2014.05.011

* Mitchison, D., Bussey, K., Touyz, S., Gonzalez-Chica, D., Musker, M., Stocks, N., Licinio, J., y Hay. P. (2019). Shared associations between histories of victimisation among people with eating disorder symptoms and higher weight. Australian y New Zealand Journal of Psychiatry, 53 (6), 540-549. https:// doi.org/10.1177/0004867418814961

Moher, D., Shamseer, L., Clarke, M., Ghersi, D., Liberati, A., Petticrew M., Shekelle, P., Stewart L. A., y PRISMA-P group. 
(2015). Preferred reporting items for systematic review and meta-analysis protocols (PRISMA-P) 2015 statement. Systematic Reviews 4, 1. https://doi.org/10.1186/2046-4053-4-1

Molendijk, M. L., Hoek, H. W., Brewerton, T. D., y Elzinga, B. M. (2017). Childhood maltreatment and eating disorder pathology: a systematic review and dose-response metaanalysis. Psychological Medicine, 4 (8) 1402-1416. https:// doi.org/10.1017/S0033291716003561

Monell, E., Clinton, D., y Birgegård, A. (2018). Emotion dysregulation and eating disorders-Associations with diagnostic presentation and key symptoms. International Journal of Eating Disorders, 51 (8), 921-930. https://doi. org/10.1002/eat.22925. PMID: 30030942

Moulton, S.J., Newman, E., Power, K., Swanson, V., y Day, K. (2015). Childhood trauma and eating psychopathology: a mediating role for dissociation and emotion dysregulation? Child Abuse y neglect, 39, 167-174. https://doi.org/10.1016/j. chiabu.2014.07.003

Neumark-Sztainer, D., Story, M., Hannan, P. J., Beuhrin., T, y Resnick, M.D. (2000). Disordered eating among adolescent: Associations with sexual/physical abuse and other familial/ psychosocial factors. International Journal of Eating Disorders, 28, 249-258. https://doi.org/10.1002/1098$108 \times(200011) 28: 3<249$ ::aid-eat1 $>3.0 . c 0 ; 2-h$

NICE. National Institute of clinical excellence. (2017). Eating disorders. Core intervention in the treatment of and management of Anorexia Nervosa, Bulimia Nervosa and related eating disorders. Guía clínica 9 Recuperado el 12/1/2019 de http://guidance.nice.org.uk.

Palmisano, G. L., Innamorati, M., Susca, G., Traetta, D., Sarracino, D., y Vanderlinden, J. (2018). Childhood Traumatic Experiences and Dissociative Phenomena in Eating Disorders: Level and Association with the Severity of Binge Eating Symptoms. Journal of Trauma y Dissociation, 19 (1), 88-107. https://doi.org/10.1080/15299732.2017.1304490.

Paxton, S. J. y McLean, S. A. (2010). Treatment for Body-Image Disturbances. In Grilo, S.M. y Mitchell, J.E. (Ed.). The treatment of eating disorders (pp 471-86). The Guilford Press.

Pignatelli, A. M., Wampers, M., Loriedo, C., Biondi, M., y Vanderlinden, J. (2017). Childhood neglect in eating disorders: A systematic review and meta-analysis. Journal of Trauma Dissociation, 18 (1), 100-115. https://doi.org/10.1080/152997 32.2016.1198951

Preti, A., Incani, E., Camboni, M. V., Petreto, D. R., y Masala, C. (2006). Sexual abuse and eating disorders symptoms: the mediator role of body dissatisfaction. Comprehensive Psychiatry, 47, 475-481. https://doi.org/10.1016/j.comppsych.2006.03.004

Quick, V. M. y Byrd-Bredbenner, C. (2014). Disordered eating, socio-cultural media influencers, body image, and psychological factors among a racially/ethnically diverse population of college women. Eating behaviors, 15 (1), 37-41. https://doi.org/ 10.1016/j.eatbeh.2013.10.005

* Richardson, A. S., Dietz, W. H. y Gordon-Larsen, P. (2014). The association between childhood sexual and physical abuse with incident adult severe obesity across 13 years of the National Longitudinal Study of Adolescent Health. Paediatric Obesity, 9 (5), 351-356. https://doi.org/10.1111/j.2047-6310.2013.00196.x

Richter, A., Gilbert, P. y McEwan, K. (2009). Development of an early memories of warmth and safeness scale and its relationship to psychopathology. Psychology and psychotherapy: theory, research and practice, 82 (2), 171-184. https://doi.org/ 10.1348/147608308X395213

* Roenholt, S., Beck, N. N., Karsberg, S. H. y Elklit, A. (2012). Posttraumatic stress symptoms and childhood abuse categories in a national representative sample for a specific age group: associations to body mass index. European Journal of psychotraumatology, 3, 17188-17194. https://doi.org/10.3402/ejpt.v3i0.17188

Rosen, J.C. (1992). Body image disorder: Definition, development, and contribution to eating disorders. In J. H. Crowther, D. L. Tennenbaum, S. E. Hobfoll, y M.A.P. Stephens (Ed.). The etiology of bulimia: The individual and familial context (pp. 157-177). Hemisphere Publishing Corporation.

Salaberria, K. Rodríguez, S., y Cruz, S. (2007). Percepción de la imagen corporal. Osasunaz, 8, 171-183. BIBLID 1577-8533.

Schore, A. N. (2003). Affect Dysregulation and Disorders of the Self. Norton y Co.

Sepúlveda, A. R., León J. A., y Botella, J. (2004). Aspectos controvertidos de la imagen corporal en los trastornos de la conducta alimentaria. Clínica y Salud, 15 (1), 55-74. https:// www.redalyc.org/articulo.oa?id=1806/18067820003

Sepúlveda, A.R., Botella, J., y León J. A. (2002). Body-image disturbance in eating disorders: a meta-analysis. Psychology in Spain, 6 (1), 83-95. https://dialnet.unirioja.es/servlet/ articulo? codigo $=297584$

Shilder P. (1935). The image and appearance of human body. International University Press.

Strauss, M. A. y Gelles, R.J. (1990). Physical Violence in American Families: Risk Factors and Adaptations to Violence in 8,145 Families. New Brunswick: Transaction Publishers.

Striegel-Moore, R. H. y Bulik, C. M. (2007). Risk factors for eating disorders. American Psychologist, 62 (3), 181-198. https://doi.org/10.1037/0003-066X.62.3.181

Striegel-Moore, R. H., Dohm, F. A., Pike, K. M., Wilfley, D. E., y Fairburn, C. G. (2002). Abuse, bullying and discrimination as risk factors for binge eating disorders. American Journal of Psychiatry, 159 (11), 1902-1907. https://doi.org/10.1176/ appi.ajp.159.11.1902

Tabri, N., Murray, H. B., Thomas, J. J., Franko, D. L., Herzog, D. B., y Eddy, K. T. (2015). Overvaluation of body shape/weight and engagement in non-compensatory weight-control behaviours in eating disorders: is there a reciprocal relationship? Psychological Medicine, 45 (14), 2951-2958. https://doi.org/10.1017/S0033291715000896

Tagay, S., Erim, Y., Stoelk, B., Möllering, A., Mewes, R., y Senf, W. (2007). The Essen Trauma-Inventory (ETI) - A screening instrument of identification of traumatic events and posttraumatic disorders. Zeitschrift für Psychotraumatologie, Psychotherapiewissenschaft, Psychologische Medizin, 1, 75-89. ht tps://pdfs.semanticscholar. org/136e/ 46f7b506be2ff59cd8f123f02049517fadeb.pdf

* Tagay, S., Schlottbohm, E., Reyes-Rodriguez, M. L., Repic, N., y Senf, W. (2014). Eating Disorders, Trauma, PTSD and Psychosocial Resources. Eating Disorders, 22 (1), 33-49. https://doi.org/10.1080/10640266.2014.857517

Tagay, S., Schlelgl, S. y Senf, W. (2010). Traumatic events, posttraumatic stress symptomatology and somatoform symptoms in eating disorder patients. European Eating Disorders Review, 18, 124-132. https://doi.org/10.1002/erv.972 
Taylor, S. E., Lerner, J. S., Sage, R. M., Lehman, B. J., y Seeman, T. E. (2004). Early environment, emotions, responses to stress, and health. Journal of Personality, 72, 1365-1393. https://doi. org/10.1111/j.1467-6494.2004.00300.x

Taylor, C. B., Bryson, S., Celio Doyle, A. A., Luce, K. H., Cunning, D., Abascal, L. B., Rockwell R., Field A. E., StriegelMoore, R., Winzelberg, A. J., y Wilfley, D. E. (2006). The adverse effect of negative comments about weight and shape from family and siblings on women at high risk for eating disorders. Pediatrics, 118 (2), 731-738. https://doi. org/10.1542/peds.2005-1806.

Thompson, K. M. y Wonderlich, S. A. (2004). Child sexual abuse and eating disorders. In J. K. Thompson (Ed). Handbook of eating disorders and obesity. (pp 679-694). Hoboken.

Tylka, T. L. y Wood-Barcalow, N. L. (2015). What is and what is not positive body image? Conceptual foundations and construct definition. Body Image, 14, 118-29. ISSN 17401445. doi: 10.1016/j.bodyim.2015.04.001.

Treasure, J., Antunes Duarte, T., y Schmidt, U. (2020). Eating disorders. Lancet, 395, 899-911. https://doi.org/10.1016/ S0140-6736(20)30059-3

Treasure, J., Leslie, M. Chamie, R., y Fernandez-Aranda, F. (2018). Are transdiagnostic models of eating disorders fit for purpose? A consideration of evidence for food addiction. European Eating Disorders Review, 26, 83-91. https://doi. org/10.1002/erv. 2578

Treurer, T., Koperdak, M., Rózsa, S., y Juredi, J. (2005). The impact of physical and sexual abuse on body image in eating disorders. European and Eating Disorder Review, 13, 106111. https://doi.org/10.1002/erv.616

Troisi, A., Di Lorenzo, G., Alcini, S., Croce Nanni, R., Di Pasquale, C., y Siracusano, A. (2006). Body Dissatisfaction in Women with Eating Disorders: Relationship to Early Separation Anxiety and Insecure Attachment. Psychosomatic Medicine, 68 (3), 449-453. https://doi.org/10.1097/01. psy.0000204923.09390.5b

Trottier, K. y MacDonald, D.E. (2017). Update on Psychological Trauma, Other Severe Adverse Experiences and Eating Disorders: State of the Research and Future Research Directions. Current Psychiatry Report, 19, 45-53. https://doi. org/10.1007/s11920-017-0806-6.

Trucco, M. (2002). Estrés y trastornos mentales, aspectos neurobiológicos y psicosociales. Revista Chilena de Neuropsiquiatría, 40 (2), 8-19. https://doi.org/10.4067/ S0717-92272002000600002.
Van den Berg, P., Paxton, S. J., Keery, H., Wall, M., Guo, J., NeumarkSztainer, D. (2007). Body dissatisfaction and body comparison with media images in males and females. Body Image, 4 (3), 257-68. https://doi.org/10.1016/j.bodyim.2007.04.003.

Van Gerko, K., Hughes, M. L., Hamill, M., y Waller, G. (2005). Reported childhood sexual abuse and eating-disordered cognitions and behaviors. Child Abuse y Neglect, 29, 375-382. https://doi.org/10.1016/j.chiabu.2004.11.002

Vaquero-Cristóbal, R., Alacid, F., Muyor, J. M., y López-Miñarro, P. A. (2013). Imagen corporal; revisión bibliográfica. Nutrición Hospitalaria, 28 (1), 27-35. https://www.redalyc.org/articulo. oa? id=3092/30922605500

* Vartanian, L. R., Hayward, L. E., Smith, J. M., Paxton, S. J., y Touyz, S. W. (2018). Risk and resiliency factors related to body dissatisfaction and disordered eating: the identity disruption model. International Journal of Eating Disorders, 51, 322-330. https://doi.org/10.1002/eat.22835

Vierling, V., Etori, S., Valenti, L., Lesage, M., Pigeyre, M., Dodin, V., Cottencin, O., y Guardia, D. (2015). Prévalence et impacte de l'état de stréss post-traumatique chez les patients atteints de trouble du comportement alimentaire. Presse Medicine, 44, e341-e352. https://doi.org/10.1016/j.lpm.2015.04.039

Villarino Marín, A. (2012). Trastornos de la conducta alimentaria. No todo es anorexia y bulimia. En Instituto Tomas Pascual Sanz (Ed). Controversias sobre los trastornos alimentarios (pp 40-43). IMC, SA.

* Villarroel, A. M., Penelo, E., Portell, M., y Raich, R. M. (2012). Childhood sexual and physical abuse in Spanish female undergraduates: does it affect eating disturbance? European Eating Disorders Review, 20, e32-e41. https://doi.org/10.1002/erv.1086

Wildes, J. E. y Marcus, M. D. (2013). Incorporating dimensions into the classification of eating disorders: three models and their implications for research and clinical practice. The International journal of eating disorders, 46 (5), 396-403. https://doi.org/10.1002/eat.22091

Wolfe, J., Kimerling, R., Brown, P. J., Chrestman, K.R., y Levin, K. (1996). Life Stressor Checklist--Revised (LSC-R). APA PsycTests. https://doi.org/10.1037/t04534-000

Wufong, E., Rhodes, P., y Conti, J. (2019). «We don’t really know what else we can do»: Parent experiences when adolescent distress persists after the Maudsley and family-based therapies for anorexia nervosa. Journal of Eating Disorders 7, 5. https:// doi.org/10.1186/s40337-019-0235-5 